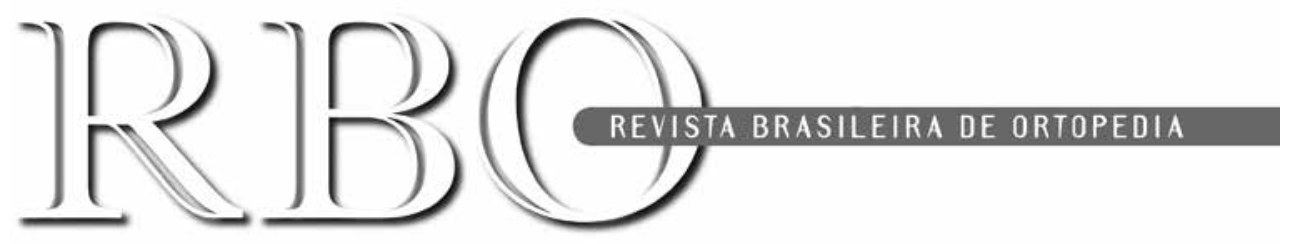

\title{
Editorial
}

\section{The difficult art of publishing}

Every day, we have hundreds of ideas, develop some and write up very few. Writing scientific articles is a difficult task and requires technique and rules so that all texts follow a single system. If not, it would be impossible to judge and store the ideas.

As editor of the $R B O$, I am very often presented with texts that demonstrate that the authors have interesting material and experience on the topic, but total lack of knowledge of the techniques and rules that guide the writing of scientific studies. Our editors very often reject these studies using the justification of lack of adequate methodology. When we try to publish in journals in other countries, we are judged harshly and it is not rare for us to give up trying to understand this difficult methodology.

How can we become familiar with this methodology, so that we can practice it correctly?

It is an exercise in learning on one's own, complemented by guidance from formerly self-taught individuals, with learning through trial and error.

The $R B O$ and the majority of journals publish such guidance, but this subject would merit a specific and constantly available course for people who want to present their experiences to the world. I have been invited by some regional associations to speak on this subject, I have gone there and there has always been interest from the audience.

Personally, I believe that for some time this difficulty was talked up by the few who had mastered this art, on order to create a degree of differentiation. These were people who spoke some mystic language.

Until such time as it is decided to establish a constantly available course, held at least once a year, let us attempt to aid those who have doubts about how to structure and write scientific papers, through a simulated example.

Let us suppose that we are going to write a paper in which we examine the preferences among homosexuals for the existing soccer teams, i.e. which soccer team homosexuals prefer.

This idea needs to be fixed in our minds. I always have the habit of writing a phrase that defines the study objective at the outset, so that when I read it again, I remember what my objective was.

This is the first step in a scientific study. In other words, to define clearly and objectively what the objectives of one's study are.

In our example, we can drift along various paths that lead us to the abysm of inclusion. We need to define our limits. Should we consider teams in a single state or in the whole country? Should we only consider declared homosexuals or also those who appear to be? Should we accept both male and female homosexuals? How is the declaration of preference or of being a fan or supporter to be defined?

This is the declaration of our material, which along with its inclusion and exclusion factors is very important. Let us therefore define, so that we can give a practical direction to our example, that we will select teams from a single state, consider people to be homosexuals if they declare themselves as such and consider the responses to the question "which team do you prefer?" (only one team). Undeclared homosexuals and metrosexuals (up to four homosexual relationships per year) will be excluded. Objectivity at this point in the study defines its quality; if we start to deviate, we will get nowhere.

The material needs to be qualified in terms of age, racial group, sex and/or profession, i.e. everything that might define one's sample. The better defined the sample is, the clearer the study will be.

Our method will be to take the responses to the direct question "which team do you prefer?" - which we will ask our defined material to answer. We will not take into consideration any comments and we will not ask any additional questions. What about the number of interviewees? Is this the size of our sample? We could calculate this through s statistical formula, or 
estimate it according to what our possibilities are, but it is essential for this number to be stated clearly so that readers can qualify the sample.

I would imagine that, because of the number of homosexuals, the statistical calculation might easily exceed tens of thousands, which would make our study impossible. Let us consider 100 cases and cite this number directly, perhaps even in the title of the study.

The authors' sensitivity and the realities of the professional setting within which they work will define the size of the sample, with its upper and lower limits. The editors will judge the appropriateness of the conclusions based on the sample presented.

With these data, we will know which teams are preferred among 100 homosexuals evaluated, and the percentages of these preferences. These will be our results.

All the steps, from the study design to the results, should be analyzed in the discussion, comparing them with the existing literature. Why analyze the soccer team preferences of homosexuals? Why define a group of homosexuals? Why one simple question? Why 100 individuals? If there is any difference in the percentage distribution, the discussion is where the analysis should be made. All these points should be answered and supported with the literature, if papers on this matter already exist.

The conclusions will be dry and defined: the preferred team among the male homosexuals in the state of xxx is team yyy.

In this example study, let us analyze what the most frequent errors made by authors are, using a sample of errors from our experience at the $R B O$.

\section{- Lack of clarity of objective}

In our example, we only want to know what the preference of the group studied is in relation to soccer teams, and not the reasons for this preference or the correlation between this preference and the qualities of the team chosen, or any other interpretation.

- Confusion between the material and the results

In our example, we use a very restricted model with few variables. Thus, the material is very well defined. We will study 100 male homosexuals, among whom one of the few variables in the group is their ages. The subjects' ages are part of the material and not the results. The division according to age can be cited, but always in the material. We may divide our results taking preferences according to age into account, and then we can cite them in the results.

- Lack of detailing in the description of the method

In our example, there is a single question with a single response from a single type of individual. The method has to be clear, since the more restricted the study is, the more useful the results will be. The description of the method cannot allow doubts.

- Greater coverage of the results that the material allows

Our result will only inform the responses given by this group of individuals. Any analysis on the team chosen, or on other teams cited, or what this preference signifies will lead us to serious errors of interpretation.

- Comments beyond what the study allows

Our example only allows us to analyze these individuals' preference regarding soccer teams. Any citation of the literature on homosexuality or on the habits of the individuals in this group has no relationship with this study and therefore must not be used.

\section{- Expanded conclusions}

We have to restrict ourselves to the question asked in the objectives of the study, and not extend our conclusions.

In our example, we wanted to know which team was preferred among 100 homosexuals and not how many homosexuals there are among each team's supporters. If the majority of the homosexuals analyzed prefer a certain team, this does not mean that, for example, this team would have the largest number of homosexual supporters in the state.

These six examples are the most common errors that we observe among authors who send their studies to the $R B O$, but other less frequent errors also occur.

If we could summarize with a single piece of advice, we would do so with the word objectivity. The fewer the number of variables considered is, the more solid our conclusions will be.

Regarding the example that we used, this study would have little or no usefulness in São Paulo, since homosexuals clearly have a preference for one particular soccer team. Perhaps in other states...? 